\title{
Early surgical intervention for structural infantile spasms in two patients under 6 months old: a case report
}

\author{
Haiyan Yang ${ }^{1+}$, Zhiquan Yang ${ }^{2 \dagger}$, Jing Peng ${ }^{1}$, Yehong Huang ${ }^{1}$, Zhuanyi Yang ${ }^{2}$, Fei Yin ${ }^{1}$ and Liwen $\mathrm{Wu}^{3^{*}}$ (D)
}

\begin{abstract}
Background: Infantile spasms (IS) are the most common childhood epileptic encephalopathy. Focal cortical dysplasia (FCD) and gray matter heterotopias $(G H)$ are common structural causes of IS. The recommended first-line treatment for IS patients with structural causes is surgical intervention, according to the International League Against Epilepsy (ILAE) commission guidelines. However, there is currently no consensus on appropriate timings of surgery.

Case presentations: Two structural IS cases are presented here: one was caused by FCD, and the other by GH. Both patients exhibited recurrent seizures at the age of 2 months, had poor responses to various antiepileptic drugs (AEDs) and displayed severe mental and motor developmental retardation. Seizure types included focal seizures and spasms. Brain magnetic resonance imaging showed abnormal gray signal or suspicious FCD lesions that coincided with the origin of the focal seizures. The patients underwent lesion resection before the age of 6 months. Follow-up observation showed that seizures of both patients were completely controlled several days after the surgery. All AEDs were gradually reduced in dosage within 1 year, and the mental and motor development almost returned to normal.
\end{abstract}

Conclusion: Early resection of lesions in structural IS patients has benefits of effectively controlling convulsions and improving developmental retardation. Infants at several months of age can well tolerate craniotomy, and their cognitive development is more likely to return to normal after early surgery.

Keywords: Infantile spasms, Focal cortical dysplasia, Gray matter heterotopias, Early lesion resection

\section{Background}

West syndrome (WS), also known as infantile spasms (IS), occurs in infancy with a peak between 4 and 7 months of age. WS has three characteristics: spasms, neurodevelopmental regression, and hypsarrhythmia on electroencephalogram (EEG) [1]. The International League Against Epilepsy (ILAE) commission classifies the etiologies of WS into genetic, structural, metabolic and unknown causes [2]. According to the ILAE guidelines, the recommended first-line treatment for IS patients with structural causes is surgical intervention [3]. In recent years, multicenter studies have shown that surgical treatment can terminate or significantly alleviate seizures in some IS patients with localized brain abnormalities [4]. However, precisely how surgical variables (i.e., timing of surgery and duration of epilepsy prior to surgery) influence neurodevelopmental outcomes remains to be determined.

\footnotetext{
* Correspondence: 271417152@qq.com

${ }^{+}$Haiyan Yang and Zhiquan Yang are co-first authors.

${ }^{3}$ Department of Neurology, Hunan Children's Hospital, Changsha 410007,

China

Full list of author information is available at the end of the article
}

\section{Case presentations}

Here, we present two IS cases. Both patients exhibited recurrent seizures including focal seizures and typical 
spasms, had no response to medical interventions, and displayed moderate to severe developmental retardation.

\section{Case 1}

This female patient exhibited recurrent seizures when she was a 50-day-old baby. Video EEG before surgery showed background hypsarrhythmia and within $15 \mathrm{~h}, 7$ seizure attacks, including 3 spasms (Fig. 1b) and 4 focal seizures starting on the left occipital region (Fig. 1c). Cerebral MRI showed a gray signal in a semiovale located in the left parietal-occipital lobe, which was considered to be the congenital heterotopic gray matter (Fig. 1a). The patient received treatment with phenobarbital, levetiracetam, vigabatrin, adrenocorticotropic hormone $(\mathrm{ACTH})$, nitrazepam and lamotrigine before surgery, but none of these antiepileptic drugs (AEDs) showed promising efficacy. At the age of 5 months, the baby patient underwent neurosurgical resection. According to the stereotactic positioning, a $2.5 \mathrm{~cm} \times 2.0$ $\mathrm{cm} \times 2.5 \mathrm{~cm}$ lesion was resected (Fig. 2a). Pathological examination results were consistent with the characteristics of gray matter heterotopias (GH) (Fig. 2b-d).

\section{Case 2}

The patient was a female infant who experienced recurrent seizures at 2 months and 25 days. Video EEG before surgery showed many sharp waves, spikes and spikeslow waves in the right central and midline areas during the interictal period; within $4 \mathrm{~h}, 3$ partial-spasm seizures occurred originating from the right hemisphere (Fig. 3b, c). Cerebral MRI revealed an abnormal lesion in the right frontal cortex, which was considered as focal cortical dysplasia (FCD) (Fig. 3a). She was treated consecutively with levetiracetam and topiramate, but showed poor effects. Considering the parents' concerns on the side effects of hormones, and the previously-reported negative response of structural IS to ACTH, ACTH was skipped and preoperative assessments were conducted when the patient was 4 months old. Intracranial EEG recording (Fig. 4a-d) during a seizure attack showed that
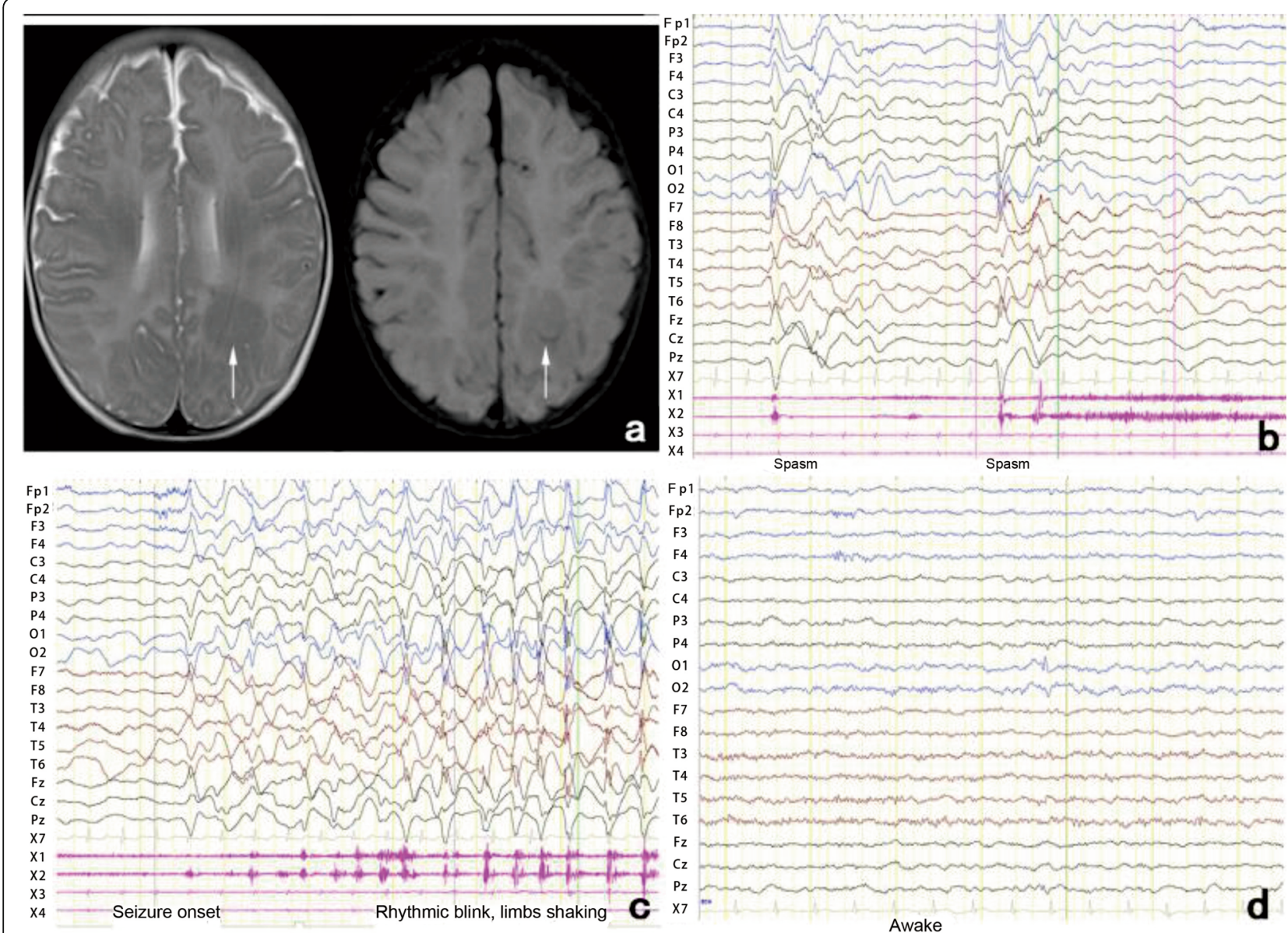

Fig. $1 \mathrm{MRI}$ and video EEG in patient 1. a Cerebral MRI revealed an abnormal gray signal (arrows) in the semiovale located in the left parietaloccipital lobe of the patient. b-d Video EEG showed spasm attacks (b), and focal seizures starting in the left occipital region (c). Normal EEG activity recorded 1 year after surgical resection $(\mathbf{d})$ 


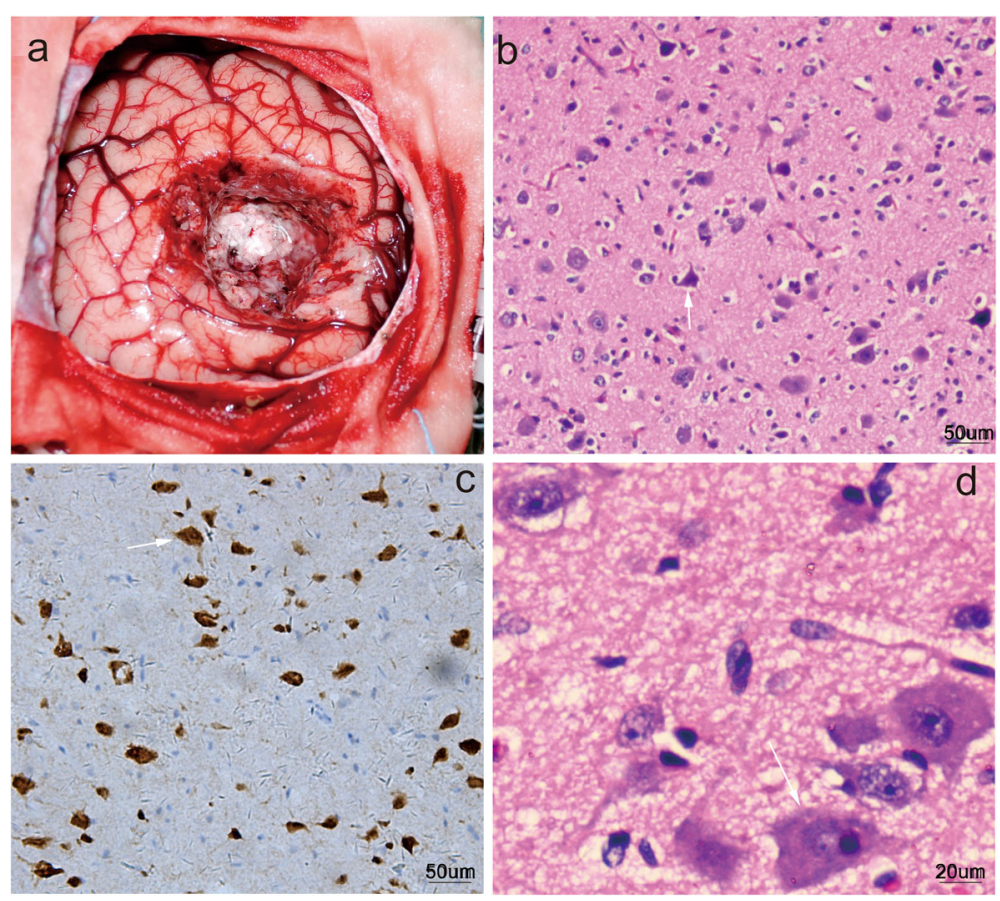

Fig. 2 Pathological examination in case 1. a Resection site. $\mathbf{b}$ HE staining of the resected tissue $(\times 200)$. c Immunohistochemical staining of NeuN (× 200). d HE staining (× 400). The pathological evaluation revealed abnormal structure and morphology of neurons, including spindle-shaped neurons, small- and medium-sized neurons and immature neurons (white arrows)

the origin site of epileptic discharge coincided with the lesion on brain MRI (Fig. 3a). Then, she underwent neurosurgical intervention. According to the stereotactic positioning, a $3 \mathrm{~cm} \times 4 \mathrm{~cm} \times 3 \mathrm{~cm}$ lesion was resected. Combined with the MRI and pathological findings, the patient was diagnosed with FCD IIa (Fig. 3a, d).

Both the baby patients had good surgical tolerability, without disturbance of consciousness and limb movement disorder. The seizures were completely controlled several days after the surgery. All AEDs were gradually reduced within 6 months, and EEG recording showed normal activity (Fig. 1d for patient 1 ). During the 5 -year follow up, both patients displayed almost normal mental and motor development.

\section{Discussion}

IS is a difficult-to-treat infantile epileptic encephalopathy by medications. Frequent seizures of IS, which can be up to hundreds of clinical and subclinical seizures per day, cause devastating effects on the brain development and cognitive levels of children, and more than $90 \%$ of patients have mental or motor developmental retardation. Some research pointed out that pediatric epilepsy surgery in infants and toddlers ( $<3$ years) offers significant opportunities for improving seizure frequency, neuro-cognitive development, and quality of life [5]. There are seven preadmission predictors for surgical candidacy: single semiology at seizure onset, structural etiology, one or more interictal foci in the same hemisphere, focal background electroencephalograph slowing, focal or hemispheric abnormality on magnetic resonance imaging, male sex, and normal development. In the presence of more than four factors, $91 \%$ were found to be surgical candidates [6]. Therefore, early surgery is recommended for those who are suitable for surgery in order to avoid intellectual and cognitive deterioration [7, 8]. The surgical treatment of IS mainly includes two methods: resection and palliative surgery. The resection surgery is preferred for patients with definite etiology, focal seizures and an origin located in the non-functional areas [4]. The palliative surgery, which mainly cuts the corpus callosum, can be used for those who have no clear etiology or manifesting widely disseminated epileptiform discharges; neuromodulation techniques such as vagus nerve stimulation (VNS) and deep brain stimulation can also be considered $[8,9]$. Delphine et al. [10] reported three such patients who presented with IS between 5 and 7 months of age; two of the patients who underwent early hemispherotomy acquired normal verbal intelligence, whereas the third patient, who was the oldest and had the longest duration of epilepsy, was operated on at 38 months of age and remained with severe mental retardation. This indicated that early diagnosis and a shorter lag time to treatment are essential for the overall outcome of WS patients [1]. At present, the earliest age for surgical treatment for IS 


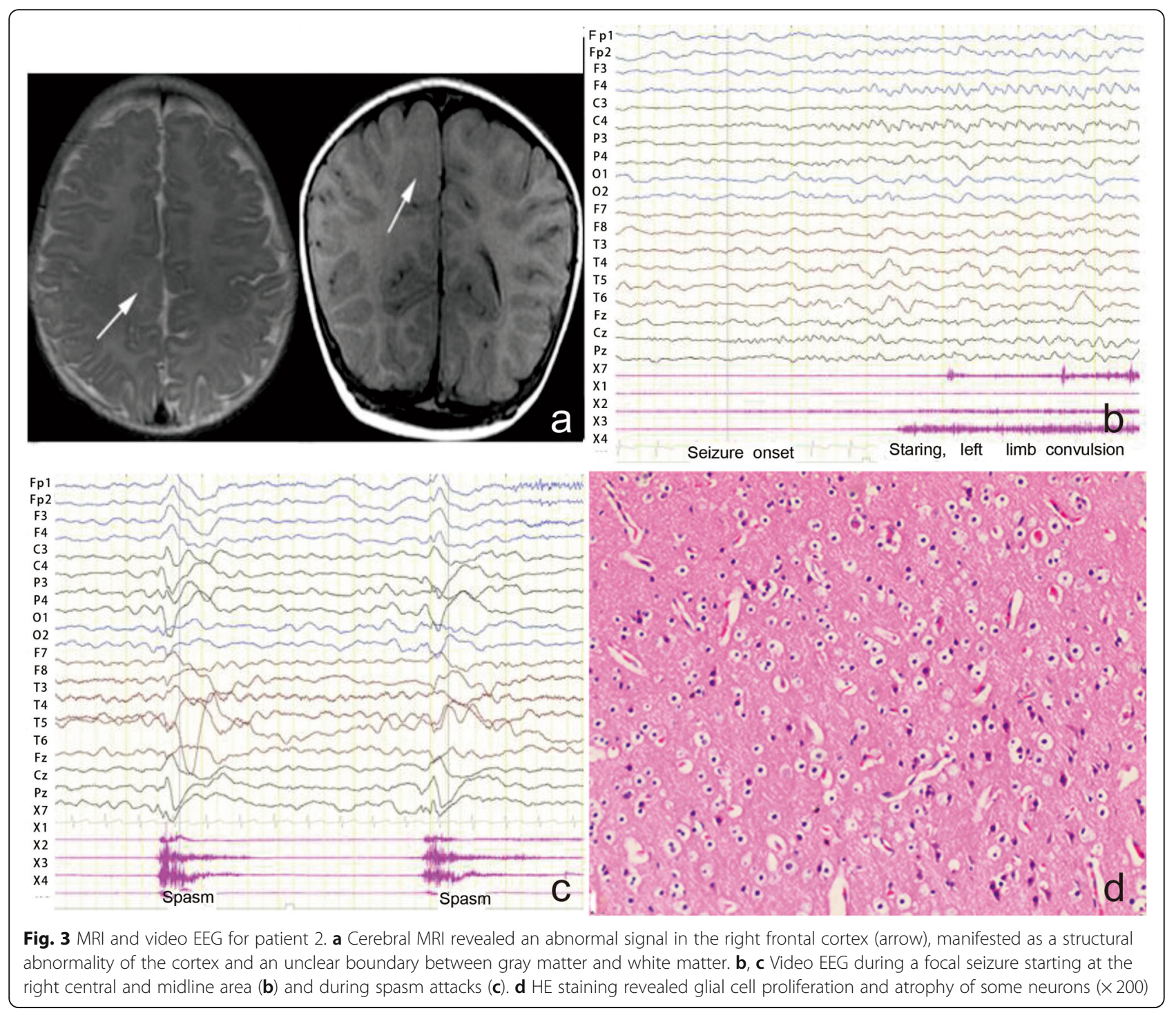

reported in the international literature is 2.5 months [11]. The earliest age for surgical treatment for IS in China is 10 months. A history of infantile spasms has been shown to be associated with a median of 3.0 years' shorter duration to surgery [12].

Kuwahara et al. [13] reported a 4-month-old female infant with atypical asymmetrical GH diagnosed with WS who underwent relatively low-dose ACTH therapy followed by a combination of valproic acid and clonazepam, and the seizures were well controlled. To date, the surgical treatment of GH-caused IS is rarely reported, and there is no consensus on how and when to treat GH combined with IS [14]. Here, we present a GH patient who manifested medically refractory epilepsy, including spasms and focal seizures, accompanied by severe developmental retardation. The patient underwent lesion resection at the age of 5 months, and showed seizure and behavioral improvement after the operation. As far as we know, this is the earliest report of neurosurgical intervention in GHinduced IS, and the patient is one of the few reported cases of lesionectomy with resultant improved behavior.

FCD is the most frequent histological finding in pediatric patients undergoing epilepsy surgery and is classified into different clinicopathological subtypes: type I, type II and type III [15]. FCD is a common structural cause of IS. Seizures from FCD are commonly refractory to medical treatment, and FCD accounts for up to $26 \%$ of pediatric epilepsy cases with surgical intervention [16]. Borggraefe et al. [17] have reported a patient who manifested FCD IIb-caused seizures within the first day of life, and received epilepsy surgery at the age of two and a half months with seizure-free outcome, suggesting that epilepsy surgery can be successfully performed in medically intractable patients with a clearly identifiable seizure onset zone within the first three months of life. This is the youngest age ever reported for surgical 

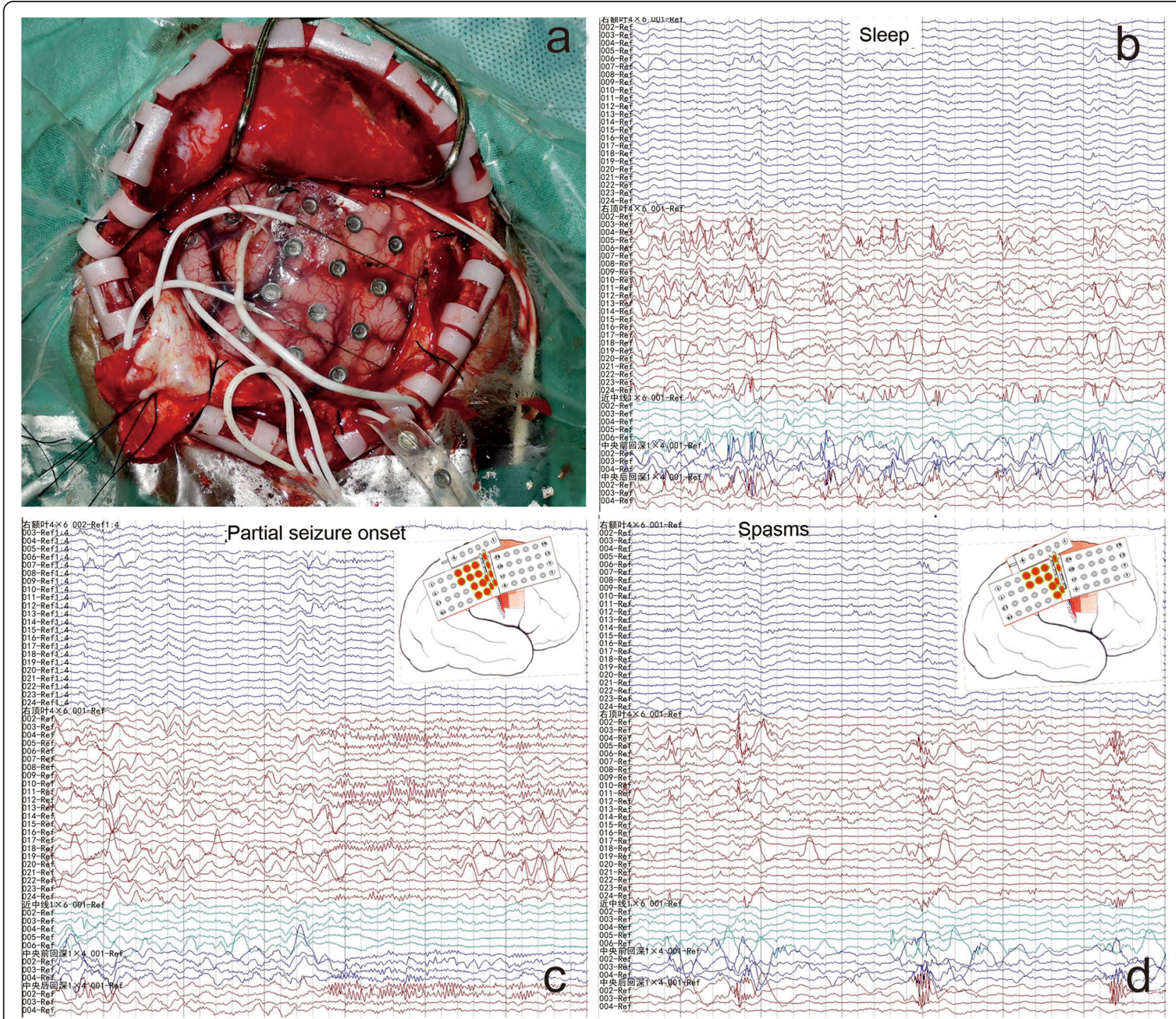

Fig. 4 Intracranial EEG for patient 2 with focal seizures and epileptic spasms. a Intraoperative electrode positioning. b A large number of focal abnormal discharges in the right parietal lobe and central area during the interictal period. c Focal seizures starting at the right central and midline area. $\mathbf{d}$ Spasm attacks

treatment of FCD-caused neonatal drug-resistant epilepsy. In addition, the report also indicated that surgical management with a shorter latency period tended to have better developmental outcomes, as demonstrated in animal models [18], in which a short duration of seizures has less influence on the near normal brain. To date, studies exploring the cognitive effects of epilepsy surgery for FCD are limited. In 2010, Rouleet-Perez et al. [19] studied 11 children who underwent epilepsy surgery before age 6. Two of the children with FCD IIb showed a rapid improvement in developmental quotient testing following surgery. Over a long-term period, only one child with FCD type IIb showed continued developmental improvement; the remaining patients were stable in their testing and did not show significant catch-up long after surgery. In this study, patient 2 was diagnosed with FCD IIa combined with IS and had a poor response to AEDs. However, after receiving neurosurgical treatment at the age of 4 months, the patient showed good surgical tolerability, rapid control of convulsions, and gradual reduction of AEDs during follow up. In addition, her mental and motor development gradually returned to normal after rehabilitation.

\section{Conclusion}

Here the two patients are very young babies with structural IS who received lesion resection surgery at early age. Their seizures were completely controlled soon after surgery, AEDs were gradually reduced, and mental and motor development almost recovered to normal levels. 
This study demonstrates that early lesion resection has more benefits for seizure control and improvement of developmental retardation. We therefore recommend that IS patients pushed to surgical intervention as early as possible for those who have single semiology at seizure onset, structural etiology, one or more interictal foci all in the same hemisphere, focal background electroencephalograph slowing, focal or hemispheric abnormality on magnetic resonance imaging, and inability of regular AEDs to effectively control seizures. This strategy will help to improve intelligence and motor development. Our case report will provide hints for pediatricians and neurosurgeons in the treatment of structural IS.

\section{Abbreviations}

ACTH: Adrenocorticotropic hormone; EEG: Electroencephalogram; FCD: Focal cortical dysplasia; GH: Gray matter heterotopias; ILAE: International League Against Epilepsy; IS: Infantile spasms; WS: West syndrome

\section{Acknowledgements}

Not applicable.

\section{Disclosures}

The authors declare that there is no conflict of interest either in financial support or relationships. Liwen Wu and Fei Yin have received research support from National Natural Science Foundation of China.

\section{Authors' contributions}

All the authors have read and approved the final manuscript.

\section{Authors' information}

Prof. Liwen Wu has been informed of all data and given her consent.

\section{Funding}

This work was supported by grants from the National Natural Science Foundation of China (No. 81671297).

\section{Availability of data and materials}

Department of Pediatrics, Department of Neurosurgery, Xiangya Hospital, Central South University.

\section{Ethics approval and consent to participate}

The use of sample from the patients was approved by the Institutional Ethics Committee of Central South University and informed consent has been obtained from patients prior to analysis.

\section{Consent for publication}

The written consent form for publication has been obtained from the guardians of patients.

\section{Competing interests}

The authors declare that they have no competing interests.

\section{Author details}

${ }^{1}$ Department of Pediatrics, Xiangya Hospital, Central South University, Changsha 410008, China. ${ }^{2}$ Department of Neurosurgery, Xiangya Hospital, Central South University, Changsha 410008, China. ${ }^{3}$ Department of Neurology, Hunan Children's Hospital, Changsha 410007, China.

Received: 16 March 2020 Accepted: 4 September 2020

Published online: 01 October 2020

\section{References}

1. D'Alonzo R, Rigante D, Mencaroni E, Esposito S. West syndrome: a review and guide for paediatricians. Clin Drug Investig. 2018;38(2):113-24.

2. Wilmshurst JM, Ibekwe RC, O'Callaghan FJK. Epileptic spasms - 175 years on: trying to teach an old dog new tricks. Seizure. 2017;44:81-6.
3. Wilmshurst JM, Gaillard WD, Vinayan KP, Tsuchida TN, Plouin P, Van Bogaert $P$, et al. Summary of recommendations for the management of infantile seizures: task force report for the ILAE Commission of Pediatrics. Epilepsia. 2015;56(8):1185-97.

4. Xu C, Yu T, Zhang G, Rajah GB, Wang Y, Li Y. Concordance between the interictal focal EEG pattern and MRI lesions as a predictor of a favorable surgical outcome in patients with epileptic spasms: a Chinese study. J Neurosurg Pediatr. 2019;23(4):422-31.

5. Pindrik J, Hoang N, Smith L, Halverson M, Wojnaroski M, McNally K, et al. Preoperative evaluation and surgical management of infants and toddlers with drug-resistant epilepsy. Neurosurg Focus. 2018;45(3):E3.

6. Haque OJ, Mandrekar J, Wyatt K, Nickels KC, Wong-Kisiel L, Wetjen N, et al. Yield and predictors of epilepsy surgery candidacy in children admitted for surgical evaluation. Pediatr Neurol. 2015;53(1):58-64.

7. Park JT, Fernandez-Baca VG, Tangen RB, Cohen ML, Miller JP. Noninvasive presurgical data for one-stage leucotomy in catastrophic epilepsy. World Neurosurg. 2018;116:268-73.

8. Abel TJ, Losito E, Ibrahim GM, Asano E, Rutka JT. Multimodal localization and surgery for epileptic spasms of focal origin: a review. Neurosurg Focus. 2018;45(3):E4

9. Baba H, Toda K, Ono T, Honda R, Baba S. Surgical and developmental outcomes of corpus callosotomy for West syndrome in patients without MRI lesions. Epilepsia. 2018;59(12):2231-9.

10. Taussig D, Dorfmuller G, Save J, Fohlen M, Chipaux M, Ferrand-Sorbets S, et al. Hemispherotomy for isolated infantile spasms following perinatal ischemic stroke. Eur J Paediatr Neurol. 2015:19(5):597-602.

11. Jazayeri MA, Jensen JN, Lew SM. Craniosynostosis following hemispherectomy in a 2.5-month-old boy with intractable epilepsy. J Neurosurg Pediatr. 2011;8(5):450-4.

12. Prideaux L, Barton S, Maixner W, Harvey AS. Potential delays in referral and assessment for epilepsy surgery in children with drug-resistant, early-onset epilepsy. Epilepsy Res. 2018;143:20-6.

13. Kuwahara M, Shima M, Nakai H, Nishino M, Fukuzumi A, Yoshioka A. A case of West syndrome with atypical massive gray matter heterotopia that is well controlled by ACTH therapy. Acta Paediatr Jpn. 1996;38(3):274-7.

14. Srour M, Rioux MF, Varga C, Lortie A, Major P, Robitaille Y, et al. The clinical spectrum of nodular heterotopias in children: report of 31 patients. Epilepsia. 2011;52(4):728-37.

15. Conti V, Pantaleo M, Barba C, Baroni G, Mei D, Buccoliero AM, et al. Focal dysplasia of the cerebral cortex and infantile spasms associated with somatic 1q21.1-q44 duplication including the AKT3 gene. Clin Genet. 2015; 88(3):241-7.

16. Gaitanis JN, Donahue J. Focal cortical dysplasia. Pediatr Neurol. 2013;49(2): 79-87.

17. Borggraefe I, Tacke M, Gerstl L, Leiz S, Coras R, Blumcke I, et al. Epilepsy surgery in the first months of life: a large type llb focal cortical dysplasia causing neonatal drug-resistant epilepsy. Epileptic Disord. 2019;21(1):122-7.

18. Wu L, Peng J, Wei C, Liu G, Wang G, Li K, et al. Characterization, using comparative proteomics, of differentially expressed proteins in the hippocampus of the mesial temporal lobe of epileptic rats following treatment with valproate. Amino Acids. 2011;40(1):221-38.

19. Roulet-Perez E, Davidoff V, Mayor-Dubois C, Maeder-Ingvar M, Seeck M, Ruffieux C, et al. Impact of severe epilepsy on development: recovery potential after successful early epilepsy surgery. Epilepsia. 2010;51:1266-76.

Ready to submit your research? Choose BMC and benefit from:

- fast, convenient online submission

- thorough peer review by experienced researchers in your field

- rapid publication on acceptance

- support for research data, including large and complex data types

- gold Open Access which fosters wider collaboration and increased citations

- maximum visibility for your research: over $100 \mathrm{M}$ website views per year

At BMC, research is always in progress.

Learn more biomedcentral.com/submission 